\title{
Minimalinvasive Therapie des Osteoidosteoms
}

Das Osteoidosteom ist ein gutartiger knochenbildender Tumor und tritt vorwiegend bei Kindern und Jugendlichen auf. Das Osteoidosteom macht etwa $10 \%$ aller gutartigen Knochen-Neoplasien aus. Er wird klinisch auffällig durch eine ausgeprägte Nachtschmerzsymptomatik. Typischerweise zeigt diese Schmerzsymptomatik auch ein deutliches Ansprechen auf die Einnah- me von Acetylsalicylsäure oder auf nichtsteroidale Antirheumatika (NSAR).

Charakteristisch ist ein strahlentransparenter Nidus, der am verlässlichsten in der Computertomografie (CT) mit Schichtdicken unter 2 mm detektiert werden kann. Der Nidus kann hierbei zentrale Verkalkungen zeigen. Der nichtverkalkte Nidus stellt sich typischerweise signalarm in T1-ge- wichteten Sequenzen und signalreich in T2-gewichteten Sequenzen der Magnetresonanztomografie (MRT) dar.

Dieser Nidus ist als der eigentliche Tumor anzusehen und in Abgrenzung zu Osteoblastomen definitionsgemäß kleiner als $1,5 \mathrm{~cm}$. Der Nidus ist zumeist von einer ausgeprägten Sklerose-Reaktion umgeben - vor allem bei intrakortikaler Lage im 

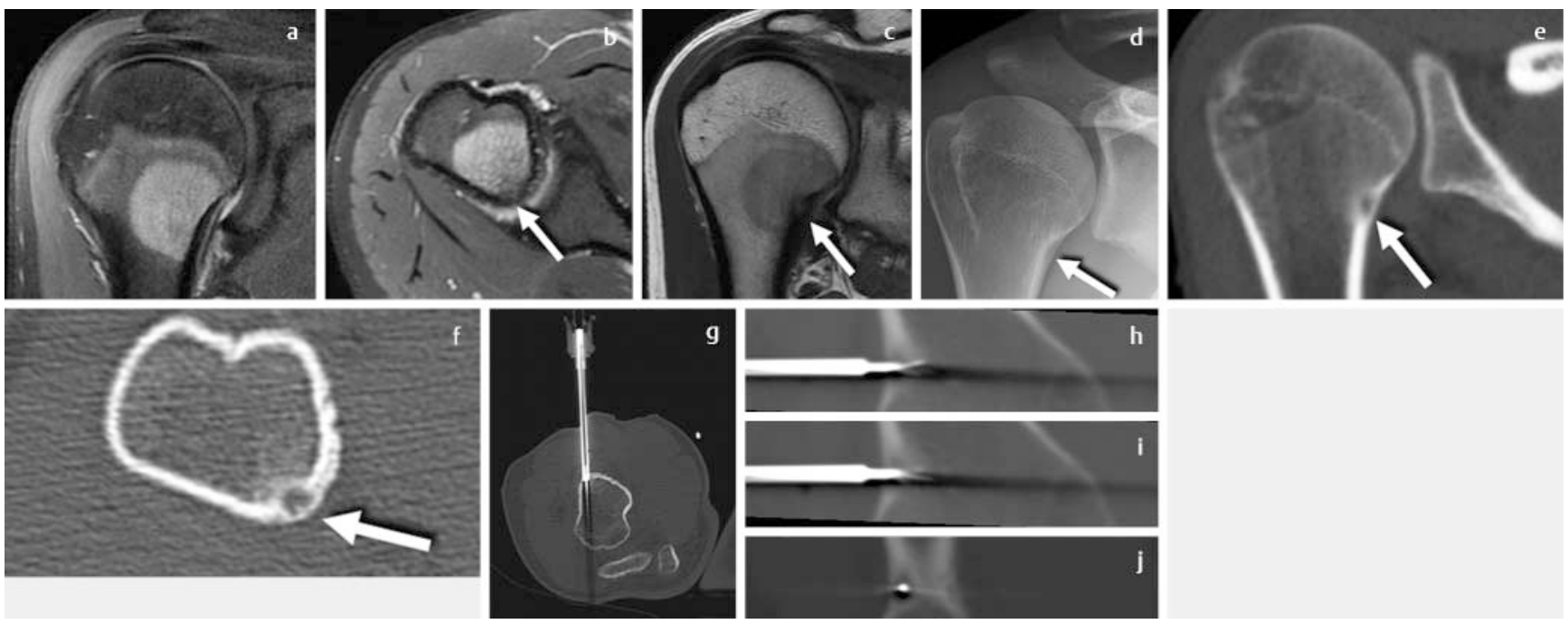

h

- Abb. 1 Komplikationslose, CT-gesteuerte Radiofrequenzablation in Vollnarkose eines Osteoidosteoms metaphysär-kortikal am medialen Collum chirurgicum des proximalen Humerus bei einer 18-jährigen Frau. A kontrastverstärkte fettsupprimierte T1-Wichtung koronar; B kontrastverstärkte fettsupprimierte T1-Wichtung axial; C koronare T1-Wichtung; D Röntgen a.-p.; E CT in koronar Reformation; F CT axial; G Zugangsweg zum Nidus; H, I RFA-Elektrode im Nidus, sagittale Ansicht; J RFA-Elektrode im Nidus, koronare Ansicht. Deutliches Knochenmarködem in der MRT (Pfeil in A), der Nidus (Pfeile) ist in der CT (E, F) besser abgrenzbar als in der MRT (B, C) und im Röntgen (D). Indikation zur RFA bei progredienter Schmerzsymptomatik mit regelmäßiger Einnahme von NSAR und einer funktionellen Einschränkung des gesamten rechten Arms bei Ausstrahlung bis in die Hand (Schmerzintensität gemäß visueller Analogskala (VAS) von 8 auf einer Skala von 0 - 10). Intervention mit direkter Punktion des Nidus bei eleviertem Arm mittels koaxialem Punktionssystem $(11 \mathrm{G} \times 6 \mathrm{~cm})$ unter CT-Kontrolle (G). Im Anschluss Einbringen der Ablationselektrode $(5 \mathrm{~mm}$ aktive Spitze) und Positionierung der aktiven Spitze im Nidus mit vorsichtigem geringem Ausfahren der Arrays (H-J). Nach Bestätigung der korrekten Lage im angestrebten Ablationsareal erfolgt die Radiofrequenzablation mit einer Temperatur von $90^{\circ} \mathrm{C}$ über eine Ablationszeit von 400 Sekunden bei 50 Watt. Am nächsten Morgen, ca. 21 h nach Intervention, gibt die Patientin keine Schmerzen mehr an (Schmerzintensität von 0 auf VAS-Skala von 0 - 10). Zudem freie Beweglichkeit des Arms.

Knochen, die aber zum Beispiel bei juxtaartikulärer oder rein auf den Markraum des Knochens beschränkter Lage auch nahezu vollständig fehlen kann. Bisweilen zeigt sich bei gelenknaher Lage eine Entkalkung der gelenknahen Knochenabschnitte und eine Synovialitis. Der Tumor verursacht trotz seiner geringen Größe starke, vor allem nächtliche Schmerzen, bedingt durch eine vermehrte Prostaglandin-Produktion im Nidus. Diese führt über eine lokale Entzündungsreaktion, Vasodilatation und erhöhte Kapillarpermeabilität zu einer Reizung afferenter Nerven und einer perifokalen, ebenfalls schmerzhaften Ödem-Bildung. Befindet sich der Tumor intraartikulär, sind die Symptome meist unspezifischer. Läsionen, die nahe der Epiphysenfuge liegen, können Wachstumsstörungen verursachen und über eine begleitende Synovialitis zu sekundären, regressiven Veränderungen des Gelenkknorpels führen. Patienten mit spinalen Läsionen entwickeln zum Teil eine reversible skoliotische Fehlhaltung. Mehr als $50 \%$ der Osteoidosteome sind in Femur und Tibia lokalisiert.
Der Nidus ist stark vaskularisiert, was sich in einer deutlichen Kontrastmittelaufnahme in der MRT äußert, die mittels Kontrastmitteldynamik gut demonstriert werden kann. Die starke Vaskularisierung des Nidus äußert sich in der Perfusionsphase der Knochenszintigrafie als zentrale Anreicherungszone, umgeben von einer weiteren Mehrspeicherung in der Spätphase im Bereich der Sklerose-Reaktion, was auch als „double density sign“ beschrieben wurde.

Differenzialdiagnostisch ist das Osteoblastom abzugrenzen, das eine dem Osteoidosteom ähnliche Morphologie und Histologie vorweisen kann und dann als eine besonders große Manifestationsform des Osteoidosteoms zu betrachten ist (benignes Osteoblastom oder Riesenosteoidosteom) mit einem Nidusdurchmesser von mehr als $1,5 \mathrm{~cm}$ bzw. als ein Osteoidosteom mit starker Wachstumstendenz. Aber trotz der histologischen Ähnlichkeit zu den Osteoidosteomen besitzen Osteoblastome zum Teil andere klinische Charakteristika (Bevorzugung der Wirbelsäule, variable Schmerzsymptomatik, geringere Kortikalis-Reaktion) und manche Osteoblastome weisen einen aggressiveren histologischen und radiologischen Aspekt auf, sodass immer eine histologische Sicherung bei diesen Tumoren angestrebt werden sollte. Des Weiteren stellt die herdförmig begrenzte Osteomyelitis, der sogenannte „Brodie-Abszess“, eine wichtige Differenzialdiagnose dar.

Therapie der Wahl des Osteoidosteoms ist die minimalinvasive Radiofrequenzablation (RFA), welche eine komplikationsarme, zielgenaue Destruktion des Tumorgewebes ermöglicht und eine exzellente klinische Erfolgsrate aufweist. Die einfache Anbohrung des Nidus mit optionaler nachfolgender Ethanol-Injektion hat eine deutlich geringere Erfolgsrate. Die chirurgische Resektion wurde von der bildgesteuerten RFA als Therapie der Wahl abgelöst. Die CT-gesteuerte, minimalinvasive RFA von Osteoidosteomen wurde erstmals im Jahre 1992 beschrieben und weist im Langzeitverlauf klinische Erfolgsraten von über $90 \%$ auf, bei durchschnittlichen Komplikationsraten von ca. $3 \%$. Bei der RFA wird hochfrequenter Wechselstrom über eine Elektrode in das Nidusgewebe appliziert. Zwischen der RFA-Sonde, die aus einem 
Metallschaft besteht, der bis auf die aktive Spitze abisoliert ist, und der Neutralelektrode kommt es so zum freien Stromfluss. Die Spannungs- und Stromdichte hat, bedingt durch die geringe Oberfläche der Sondenspitze, an der aktiven RFA-Elektrode ihr Maximum und verursacht dort eine Gewebserwärmung bis zur Nekrose. Die Größe des ellipsoiden Ablationsareals kann dabei abhängig von der gewählten aktiven Spitze über folgende Formel abgeschätzt werden:

- Längsachse: a=2 × Länge der aktiven Spitze

- Querachse: b=2/3 $\times$ Längsachse a

Die RFA erfolgt im Regelfall in Vollnarkose und wird unter CT-Kontrolle unter Verwendung dünnschichtiger NiedrigdosisProtokolle durchgeführt. Der Zugangsweg wird möglichst rechtwinklig zur Knochenoberfläche geplant, um ein Abgleiten der Knochenstanze oder des Bohrers zu verhindern. Je nach Lage der Läsion wird zur Schonung verletzlicher Gefäß- oder Nervenstrukturen ein Zugangsweg durch die Gegenkortikalis bevorzugt; bei gelenknaher Lage ist ein extraartikulärer Zugang zu wählen.

Durch Nutzung thermaler Protektionstechniken wie der Applikation von Luft in den Epiduralraum zur Vergrößerung des Abstands zwischen Rückenmark bzw. Spinalnerven und dem Nidus, dem Einsatz einer 3D-Zugangsplanung und dem Einsatz multipler Ablationspositionen sowie Temperatursensoren ist inzwischen die Ablation von Tumoren in der Wirbelsäule mit engem Lagebezug zu Nerven oder dem Spinalkanal und Nidusdurchmessern von über $2 \mathrm{~cm}$ möglich. Ist der Nidus spindelförmig bzw. überschreitet der Durchmesser 1 Zentimeter, empfiehlt sich eine Ablation in mehreren überlappenden Sondenpositionen (in domo werden aktive Spitzen der RFA-Sonde von $7 \mathrm{~mm}$ und $10 \mathrm{~mm}$ verwendet), da erfahrungsgemäß auch geringe Volumina noch vitalen $\mathrm{Ni}$ dusgewebes ein Rezidiv oder persistierende Schmerzen zur Folge haben können. Die Ablation an sich erfolgt dann in domo jeweils über 400 Sekunden mit einer Zieltemperatur von 90 Grad Celsius. Eine Ablation des Punktionstraktes ist aufgrund der Benignität des Osteoidosteoms nicht notwendig. Die in der Literatur am häufigsten beschriebene Komplikation ist die Verbrennung der Haut um die Punktionsstelle bei relativ oberflächlich gelegenen Osteoidosteomen oder aber auch an der Neutralelektrode. Eine weitere nennenswerte Komplikation sind postinterventionelle Frakturen um den Bohrkanal; daher empfehlen wir bis zu 6 Wochen Sportkarenz nach RFA von Röhrenknochen, die das Körpergewicht tragen.

Andere minimalinvasive Verfahren zur Behandlung des Osteoidosteoms, wie die Laserablation, die Mikrowellenablation, der hochintensive fokussierte Ultraschall und die Kryoablation, haben sich bisher nicht als Therapie der Wahl etablieren können.

Zusammengefasst lässt sich festhalten, dass das Osteoidosteom als gutartiger knochenbildender Tumor vorwiegend Kinder und Jugendliche betrifft und sich durch ausgeprägte Nachtschmerzen ma- nifestiert. Die Diagnosestellung ist durch den Nachweis des hypodensen Nidus innerhalb einer hyperdensen SkleroseWolke mittels CT und einer starken Kontrastmittelaufnahme im Nidus mittels dynamischer MRT meist problemlos möglich. Therapie der Wahl ist die RFA ( $\triangleright$ Abb. 1), die eine komplikationsarme und sichere Behandlung ermöglicht.

\section{Interessenkonflikt}

Die Autoren geben an, dass kein Interessenkonflikt besteht.

\section{Autorinnen/Autoren}

Marc-André Weber

Institut für Diagnostische und Interventionelle Radiologie, Kinder- und Neuroradiologie,

Universitätsmedizin Rostock

\section{Korrespondenzadresse}

Prof. Dr. med. Marc-André Weber, M.Sc. Institut für Diagnostische und

Interventionelle Radiologie, Kinder- und

Neuroradiologie

Universitätsmedizin Rostock

Ernst-Heydemann-Str. 6

18057 Rostock

marc-andre.weber@med.uni-rostock.de

Bibliografie

DOI https://doi.org/10.1055/a-0943-1561

Online-Publikation: 2019

Fortschr Röntgenstr 2019; 191: S127-S129

(c) Georg Thieme Verlag KG, Stuttgart · New York ISSN 1433-5972 